\title{
Yeast Fermentate Prebiotic Ameliorates Allergic Asthma, Associating with Inhibiting Inflammation and Reducing Oxidative Stress Level through Suppressing Autophagy
}

\author{
Subo Gong, ${ }^{1}$ Xiaoying Ji, ${ }^{2}$ Jing Su, ${ }^{1}$ Yina Wang, ${ }^{1}$ Xianghong Yan, ${ }^{3}$ Guyi Wang, ${ }^{1}$ Bin Xiao, ${ }^{1}$ \\ Haiyun Dong, ${ }^{1}$ Xudong Xiang, ${ }^{1}$ and Shaokun Liu $\oplus^{1}$ \\ ${ }^{1}$ Second Xiangya Hospital of Central South University, No. 139 Middle Renmin Road, Changsha City, Hunan Province, China \\ ${ }^{2}$ Pulmonary and Critical Care Medicine, The Seventh Affiliated Hospital, Sun Yat-sen University, No. 628, Zhenyuan Road, \\ Xinhu Street, Guangming District, Shenzhen City, China \\ ${ }^{3}$ Department of Laboratory Medicine, Changsha Central Hospital, Nanhua University, Changsha City, Hunan Province, China
}

Correspondence should be addressed to Shaokun Liu; shaokunliu228@csu.edu.cn

Received 30 July 2020; Revised 24 November 2020; Accepted 30 November 2020; Published 20 January 2021

Academic Editor: Shuai Chen

Copyright (c) 2021 Subo Gong et al. This is an open access article distributed under the Creative Commons Attribution License, which permits unrestricted use, distribution, and reproduction in any medium, provided the original work is properly cited.

\begin{abstract}
Background and Purpose. Allergic asthma, a respiratory disease with high morbidity and mortality, is reported to be related to the airway allergic inflammation and autophagy-induced oxidative stress. Although the therapeutic effects of fermentate prebiotic (YFP) on allergic asthma have been widely claimed, the underlying mechanism is still unclear. This study is aimed at investigating the possible mechanism for the antiasthma property of YFP in a mouse model. Methods. Ovalbumin was used to induce allergic asthma following administration of YFP for one week in mice, to collect the lung tissues, bronchoalveolar lavage fluid (BLFA), and feces. The pathological state, tight-junction proteins, inflammatory and oxidative stress-associated biomarkers, and TLRs/NF- $\kappa$ B signaling pathway of the lung tissues were evaluated by HE staining, immunofluorescence, ELISA, and WB, separately. RT-PCR was used to test oxidative stress-associated genes. Leukocyte counts of BLFA and intestinal microbiota were also analyzed using a hemocytometer and $16 \mathrm{~S}$ rDNA-sequencing, separately. Result. YFP ameliorated the lung injury of the mouse asthma model by inhibiting peribronchial and perivascular infiltrations of eosinophils and increasing tight-junction protein expression. YFP inhibited the decrease in the number of BALF leukocytes and expression of inflammatory-related genes and reversed OVA-induced TLRs/NF- $\kappa \mathrm{B}$ signaling pathway activation. YFP ameliorated the level of oxidative stress in the lung of the mouse asthma model by inhibiting MDA and promoting the protein level of GSH-PX, SOD, CAT, and oxidative-related genes. ATG5, Beclin1, and LC3BII/I were significantly upregulated in asthma mice, which were greatly suppressed by the introduction of YFP, indicating that YFP ameliorated the autophagy in the lung of the mouse asthma model. Lastly, the distribution of bacterial species was slightly changed by YFP in asthma mice, with a significant difference in the relative abundance of 6 major bacterial species between the asthma and YFP groups. Conclusion. Our research showed that YFP might exert antiasthmatic effects by inhibiting airway allergic inflammation and oxidative stress level through suppressing autophagy.
\end{abstract}

\section{Introduction}

Allergic asthma is a common chronic inflammatory respiratory disease with high morbidity and mortality all over the world. According to the World Health Organization (WHO) report in 2015 [1], approximately 0.3 billion patients are diagnosed with allergic asthma. Allergic asthma is mainly clinically characterized by discontinuous reversible airway obstruction and bronchial hyperresponsiveness [2]. Although numerous investigations have explored allergic asthma's pathogenesis in the past decades, allergic asthma's aetiology and pathogenesis remain unknown, which prevents pharmaceutical companies from developing effective targeted drugs and the clinicians from diagnosing accurately [3].

Airway allergic inflammation is regarded as one of the leading theories on allergic asthma [4]. As allergic asthma 
develops, large amounts of inflammatory cells infiltrate into the lung tissues in the pathological biopsy of both clinical allergic asthma patients and the experimental animal models, including granulocytes [5], mastocytes [6], macrophages [7], dendritic cells [8], and T cells and B cells [9]. The NF- $\kappa \mathrm{B}$ signal pathway is reported to be both involved in inflammatory activation, including TLR4/NF- $\kappa \mathrm{B}$ [10], CX3CR1/NF$\kappa \mathrm{B}[11], \mathrm{p} 120 / \mathrm{NF}-\kappa \mathrm{B}[12]$, and TRAF6/NF- $\kappa \mathrm{B}$ [13] signal pathways. Hong et al. [14] reported that bromodomaincontaining protein 4 inhibition alleviated matrix degradation by enhancing autophagy and suppressing NLRP3 inflammasome activity through regulating $\mathrm{NF}-\kappa \mathrm{B}$ signaling in nucleus pulposus cells. Besides, Qi et al. [15] reported that MSTN attenuated cardiac hypertrophy through the inhibition of excessive cardiac autophagy by blocking AMPK/mTOR and miR-128/PPAR $\gamma / \mathrm{NF}-\kappa \mathrm{B}$ signal pathways. These studies reveal the critical roles of $\mathrm{NF}-\kappa \mathrm{B}$ for the regulation of inflammations.

Furthermore, the mice with knockout of autophagy gene ATG5 in dendritic cells are more susceptible to steroltolerant neutrophilic airway inflammation [16], indicating that autophagy might be involved in the development and progressing of allergic asthma. Autophagy is a relatively conservative degradation of cellular materials, such as damaged organelles or reactive oxygen species (ROS) [17]. Recent studies reveal the high correlation of the nucleotide polymorphism of ATG5/7 and the development of asthma in pediatrics [18] and adults [19]. Besides, more autophagic vacuoles are observed in the clinical-pathological biopsy of allergic asthma patients [20]. Excessive production of ROS induced by the aggravation of autophagy in the tissues further contributes to oxidative stress, while oxidative stress could be suppressed by 3-MA, an autophagy inhibitor, in a murine allergic asthma mouse model [21]. Poon et al. also reported an important role of autophagy-regulated oxidative stress in the developing and processing of asthma [20]. Therefore, investigations on oxidative stress induced by autophagy should help understand allergic asthma's pathogenesis better.

Yeast Fermentate Prebiotics (YFP), a group of live microorganisms, have benefit roles in maintaining the health of the host [22], which could be associated with balancing the microbial community structure, inducing the degradation of antigens [23], and regulating immunity [24]. Recently, YFP was reported to exert therapeutic effects on the treatment of allergic asthma [25-29]. Besides, prebiotic was reported to regulate the NF- $\kappa \mathrm{B}$ signal pathway in colitis [30] and diabetes [31] by modulating gut microbiota. In the present study, the antiasthma effects of YFP and the underlying mechanism will be investigated to provide the fundamental basis for the potential therapeutic application of YFP against allergic asthma.

\section{Materials and Methods}

2.1. Animals and Allergic Asthma Model. Twenty-four 6week old BABL/c male mice were purchased from Beijing Vital River Laboratory Animal Technology Co., Ltd. The mice were divided into three groups: the control group, the asthma group, and the asthma+YFP group. The mice in the
asthma+YFP group were orally administrated with YFP $\left(1 \times 10^{9} \mathrm{CFU} /\right.$ day $)$ from day 0 to day 6 , while the other mice received oral administration of normal saline. Ovalbumin (OVA) was used to establish the murine asthma model according to a modification of the methods proposed by $\mathrm{Yu}$ et al. [29]. Briefly, the mice in the asthma group and the asthma+YFP group were administrated with an intraperitoneal injection of $20 \mu \mathrm{g}$ OVA emulsified in $2.25 \mathrm{mg}$ alum hydroxide in a total volume of $100 \mu \mathrm{L}$ at day 7 and day 14 and inhaled with 1\% OVA through an ultrasonic sprayer (Nescosonic UN-511, Alfresa, Osaka, Japan) for three days from day 21. Normal saline was administered orally instead of OVA to the control mice. On day 23, all the mice were sacrificed for the collection of lung and feces. The lungs were flushed twice with cold $0.5 \%$ fetal bovine serum in $1 \mathrm{~mL}$ PBS, and bronchoalveolar lavage fluid (BALF) was obtained for leukocyte counts using a hemocytometer (Thermo) after lavage and centrifuged at $2000 \mathrm{~g}$ at $4^{\circ} \mathrm{C}$ for $5 \mathrm{~min}$. We declare that all animal experiments involved in this manuscript were authorized by the ethical committee of The Second Xiangya Hospital of Central South University and carried out according to the guidelines for care and use of laboratory animals as well as to the principles of laboratory animal care and protection.

2.2. Enzyme-Linked Immunosorbent Assay (ELISA). Inflammatory or oxidative stress biomarkers, including tumor necrosis factor- $\alpha$ (TNF- $\alpha$ ), interleukin- (IL-) $1 \beta$, IL-6, transforming growth factor- (TGF-) $\beta$, interferon- (IFN-) $\gamma$, malondialdehyde (MDA), glutathione peroxidase (GSH$\mathrm{Px})$, superoxide dismutase (SOD), and catalase (CAT), in BALF were detected by ELISA according to the instruction of the manufacturer (Sigma-Aldrich, Missouri, USA). The samples were firstly incubated with $1 \%$ BSA and then incubated with the primary antibodies for one hour. Subsequently, the samples were mixed with streptavidin-horseradish peroxidase (HRP) conjugated secondary antibodies for 20 mins at room temperature; then, the absorption at $450 \mathrm{~nm}$ was analyzed using a microplate spectrophotometer (Thermo Fisher, Massachusetts, USA).

2.3. Hematoxylin and Eosin (HE) Staining. The lungs were washed over by sterile water for three hours, dehydrated by $70 \%, 80 \%$, and $90 \%$ ethanol solution successively, and mixed with equal quality of ethanol and xylene. After $15 \mathrm{~min}$ incubation, the tissues were mixed with equal quality of xylene for $15 \mathrm{~min}$. Repeat the steps until the tissues looked transparent. Subsequently, the tissues were embedded in paraffin, sectioned, and stained with hematoxylin and eosin (HE). $\mathrm{H} \& \mathrm{E}$-stained tissue sections were analyzed under a microscope (Olympus).

2.4. Real-Time Polymerase Chain Reaction (RT-PCR). Total RNA of the lungs was extracted using a TaKaRa MiniBEST Universal RNA Extraction Kit (TaKaRa, Dalian, China), according to the manufacturer's instructions, and quantified with a NanoDrop spectrophotometer (NanoDrop Technologies, Wilmington, DE). Complementary DNA was generated with a specific RT primer. RT-PCR was performed with SYBR Premix Ex TaqTM (Tli RNaseH plus) (TaKaRa, 
Dalian, China) by the Applied Bio-Rad CFX96 Sequence Detection System (Applied Biosystems). The expression level of GPX1-4, CAT, SOD1, SOD2, and UCP2 was defined from the threshold cycle $(\mathrm{Ct})$, and relative expression levels were calculated using the $2^{-\Delta \Delta \mathrm{Ct}}$ method after normalization regarding the expression of U6 small nuclear RNA. The expression level of GAPDH in the tissues was taken as the negative control. Three independent assays were performed. The information of the primers is shown in Table 1.

2.5. Immunofluorescence. The BALF cells were incubated with primary rabbit anti-ZO-1, anti-Claudin1, anti-Claudin4, and anti-Occludin (OmnimAbs, 1:1000) antibody overnight at $4^{\circ} \mathrm{C}$. Following washed three times with PBS, cells were incubated with secondary Cy3-conjugated antirabbit IgG (Abcam, 1:200) for $30 \mathrm{~min}$ at room temperature. The DAPI was added to dye the nuclear for $5 \mathrm{~min}$, and $50 \%$ glycerin was used to block the medium. Stained cells were photographed under a fluorescence microscope (Olympus, Tokyo, Japan).

2.6. Western Blot. Inflammatory and autophagy-related proteins were evaluated by Western blot. Proteins were extracted from the lung tissues using the Nuclear and Cytoplasmic Protein Extraction Kit (Beyotime, China). Approximately $40 \mu \mathrm{g}$ of protein was loaded and separated with the $12 \%$ SDS-polyacrylamide gel (SDS-PAGE) and then transferred to polyvinylidene difluoride (PVDF) membrane (Millipore, MIT, USA). The membrane was incubated with $5 \%$ nonfat dry milk in TBST (Tris-buffered saline/0.1\% Tween-20, $\mathrm{pH}$ 7.4) for $1 \mathrm{~h}$ at room temperature, followed by incubation overnight with primary rabbit anti-mouse antibodies to NF$\kappa \mathrm{B}(1: 1000, \mathrm{Abcam}, \mathrm{USA}), \mathrm{p}-\mathrm{NF}-\kappa \mathrm{B}(1: 1000, \mathrm{Abcam}, \mathrm{USA})$, TLR1 ( $1: 1000$, Abcam, USA), TLR2 ( $1: 1000$, Abcam, USA), TLR3 ( $1: 1000$, Abcam, USA), TLR4 ( $1: 1000$, Abcam, USA), Myd88 (1:1000, Abcam, USA), ATG5 (1:1000, Abcam, USA), LC3I/II ( $1: 1000$, Abcam, USA), Beclin $1(1: 1000$, Abcam, USA), and GAPDH (1:1000, Abcam, USA). A horseradish peroxidase-conjugated antibody against rabbit IgG (1:5000, Abcam, USA) was used as a secondary antibody. Blots were incubated with the ECL reagents (Beyetime, Jiangsu Province, China) and exposed to Tanon 5200-multi to detect protein expression. Three independent assays were performed.

2.6.1. 16S rDNA-Sequencing Analysis. Three feces were collected from each group for the microbiome analysis. Bacterial genomic DNA was extracted from feces using the Qiagen DNA Mini Kit (Qiagen, Valencia, California) according to manufacturer's protocols, and the V4-V5 hypervariable regions of the $16 \mathrm{~S}$ rDNA gene were PCR-amplified with the appropriate controls against reagent contamination. Amplified DNA fragments were sequenced using the 454 Genome Sequencer FLX platform (454 Life Sciences, Roche Diagnostics, Burgess Hill, United Kingdom). The raw data were processed using quantitative insights into microbial ecology (QIIME) pipeline version 1.7. Stringent criteria were used to remove low quality and chimeric reads. The remaining rarified reads were subject to open reference operational
TABLE 1: The primers used in this study.

\begin{tabular}{lc}
\hline Primer name & Primer sequence $\left(5^{\prime}-3^{\prime}\right)$ \\
\hline GPX1 forward & CAGTTGCAGTGCTGCTGTCTC \\
GPX1 reverse & GCTGACACCCGGCACTTTATTAG \\
GPX2 forward & GACACGAGGAAACCGAAGCA \\
GPX2 reverse & GGCCCT TCACAACGTCT \\
GPX3 forward & CTTCCTA CCCTCAAGTATGTCCG \\
GPX3 reverse & GAGGTGGGAGGACAGGAGTT CTT \\
GPX4 forward & GCAACCAGTTTGGGAGGCAGGAG \\
GPX4 reverse & CCTCCATGGGACCATAGCGC TTC \\
CAT forward & GGTCATGCATTTAATCAGGCAGAA \\
CAT reverse & TTGCTTGGGTCGAAGGCTATC \\
SOD1 forward & GAAGGTGTGGGGAAGCATTA \\
SOD1 reverse & ACATTGCCCAAGTCTCCAAC \\
SOD2 forward & CCAAATCAGGATCCACTGCAA \\
SOD2 reverse & CAGCATAACGATCGTGGTTTACTT \\
UCP2 forward & CTACAAGACCATTGCACGAGAGG \\
UCP2 reverse & AGCTGCTCATAGGTGACAAACAT \\
GAPDH forward & CAATGACCCCTTCATTGACC \\
GAPDH reverse & GAGAAGCTTCCCGTTCTCAG \\
\hline
\end{tabular}

taxonomic unit (OTU) picking (97\% identity cutoff). The mean sequencing depth used for analysis was 31615 . There is no sample drop-off. The sequence data were deposited to NCBI SRA (Sequence Read Archive) (SRP065072).

2.7. Statistical Analysis. GraphPad Prism 7.0 (GraphPad Software, USA) was employed to perform statistical analysis. Results were statistically analyzed with Student's $t$-test for two-group comparisons. Data are presented as mean \pm SEM. $P$ values $<0.05$ were considered significant.

\section{Results}

3.1. YFP Ameliorated the Lung Injury of the Mouse Asthma Model. HE staining was used to check the pathological state of the lung tissues, and the immunofluorescence assay was used to determine the expression level of tight junctionrelated proteins. As shown in Figure 1(a), significant peribronchial and perivascular infiltrations of eosinophils were observed in the asthma group, compared to the control, which were significantly suppressed by YFP. ZO-1, Claudin1, Claudin4, and Occludin were significantly downregulated in the asthma group, compared to the control, the expression of which was significantly promoted by the treatment of YFP (Figures 1(b)-1(d)).

3.2. YFP Inhibited the Inflammation in the Lung of the Mouse Asthma Model. YFP inhibited the decrease in the number of BALF leukocytes induced by OVA (Figure 2(a)). As shown in Figures 2(b)-2(f), the concentration of TNF- $\alpha$, IL- $1 \beta$, IL- 6 , TGF- $\beta$, and IFN- $\gamma$ was significantly elevated in the asthma group compared to the control group, which was suppressed by the introduction of YFP. To further evaluate the effects of 


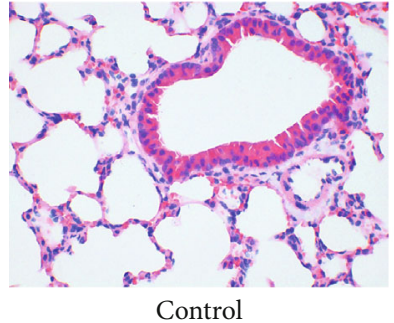

Control

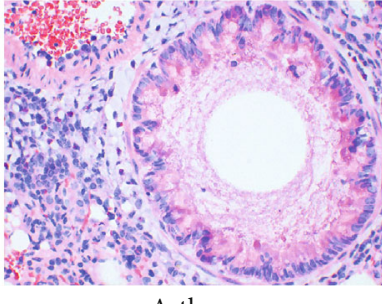

(a)

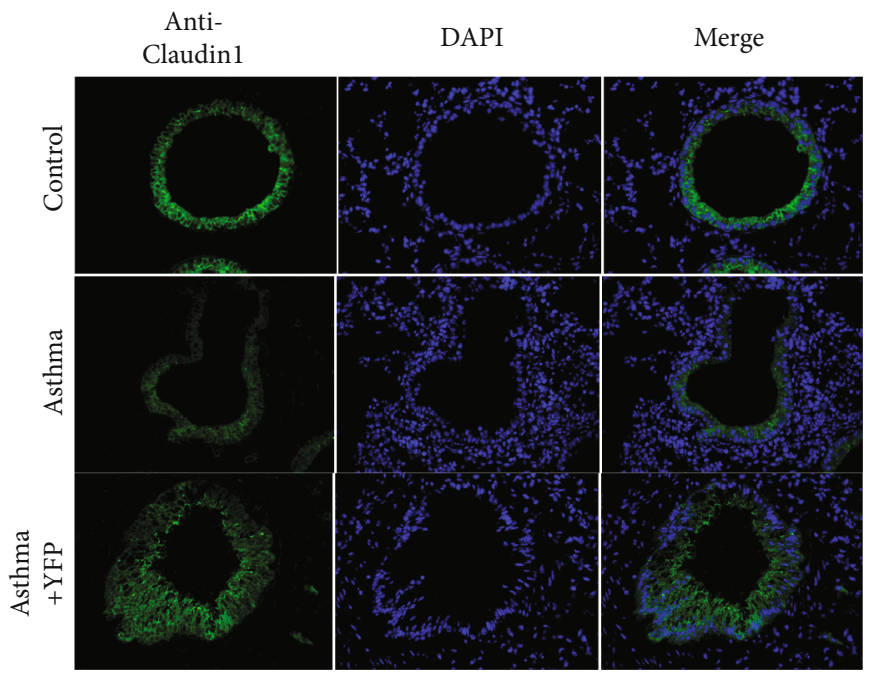

(b)

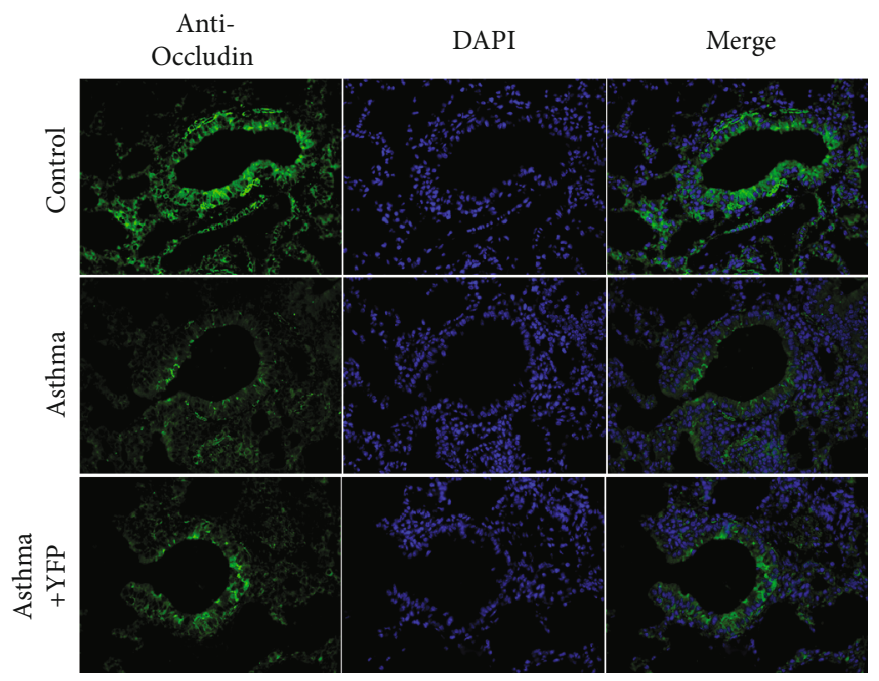

(d)

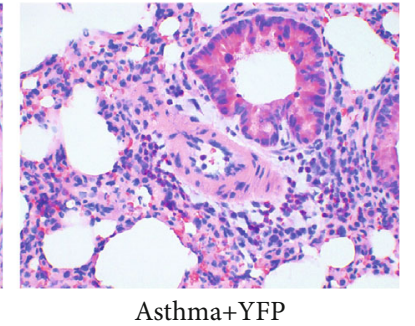

Asthma+YFP

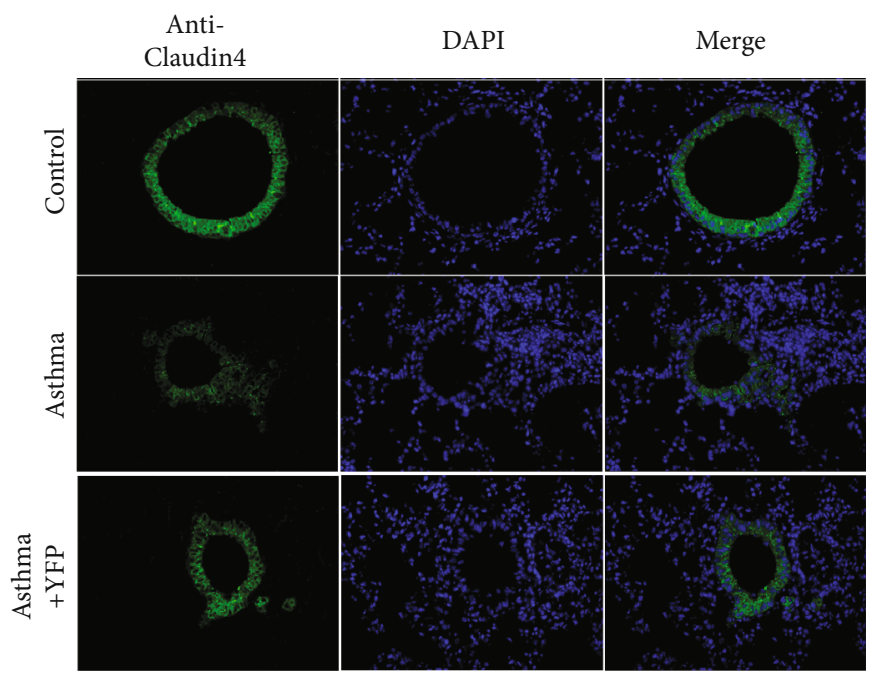

(c)

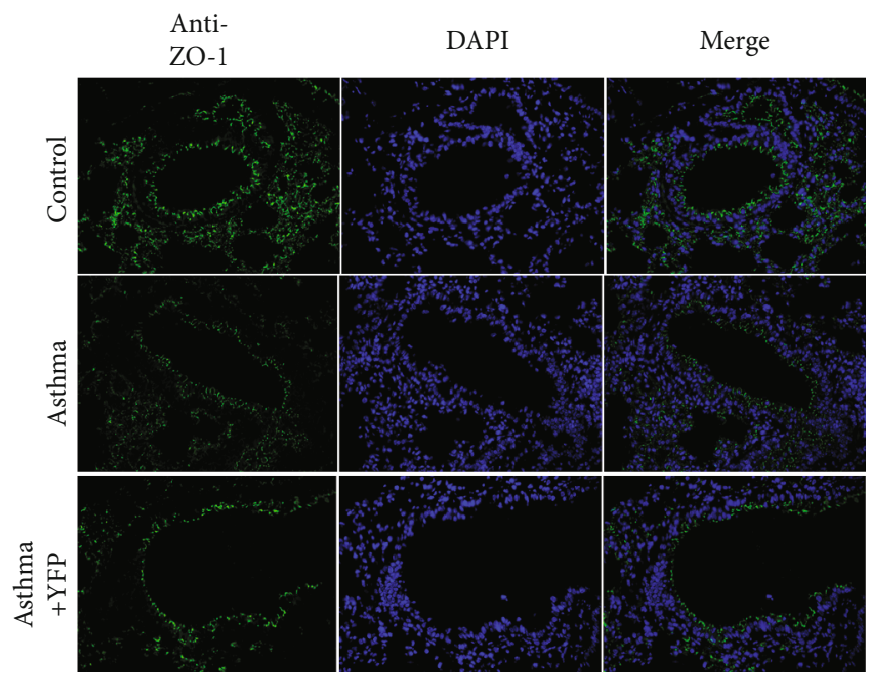

(e)

FIGURE 1: YFP ameliorated the injury of lung tissue of the mouse asthma model. HE staining of the lung tissue from each mouse (a). The expression of Claudin1 (b), Claudin4 (c), Occludin (d), and ZO-1 (e) was detected by immunofluorescence.

YFP on the inflammation, the expression of related proteins in the lung tissue was detected by Western blot. As shown in Figures 2(g) and 2(h), the expression level of NF- $\kappa \mathrm{B}, \mathrm{p}-$ NF- $\kappa \mathrm{B}$, TLR1, TLR, TLR3, TLR4, and Myd88 was significantly promoted in the asthma group, compared with the control group, which was also inhibited by the treatment with YFP $\left({ }^{* *} P<0.01\right.$ vs. control, ${ }^{* * *} P<0.001$ vs. control, and ${ }^{\# \# \#} P<0.001$ vs. asthma).
3.3. YFP Ameliorated the Level of Oxidative Stress in the Lung of the Mouse Asthma Model. Oxidative stress-related factors were detected in the BALF by ELISA to evaluate the effect of YFP on oxidative stress. Compared to the controls, MDA was significantly improved in the asthma group, which was inhibited by the introduction of YFP (Figure 3(a)), while the decreased production of GSH-PX, SOD, and CAT in the asthma group was significantly elevated by the treatment 


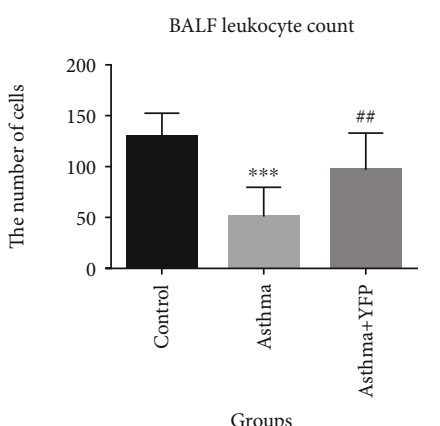

(a)

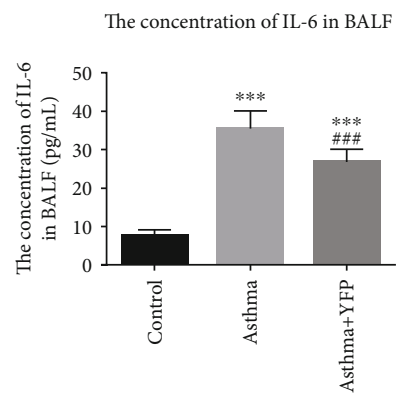

Groups

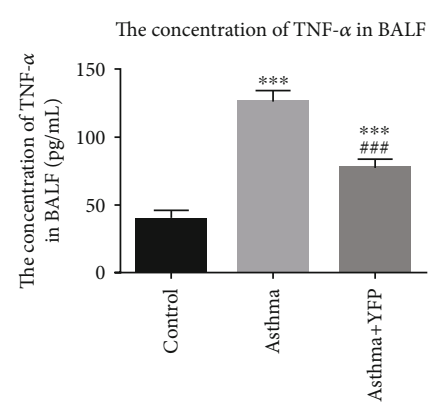

(b)

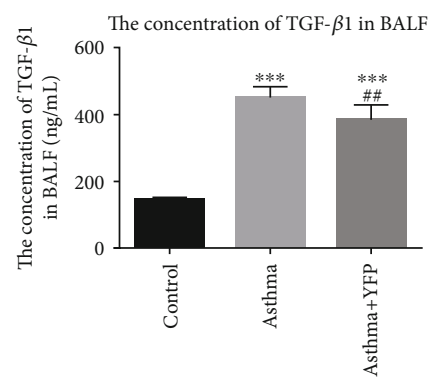

Groups

(e)

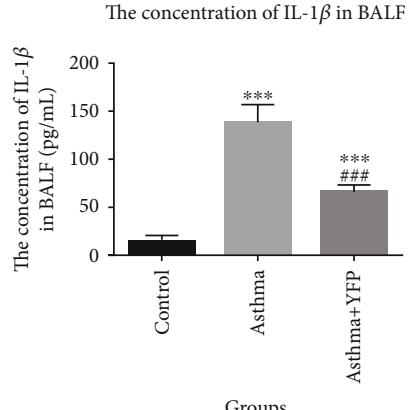

(c)

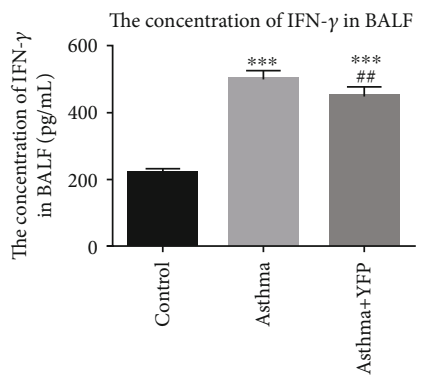

Groups

(d)

(f)

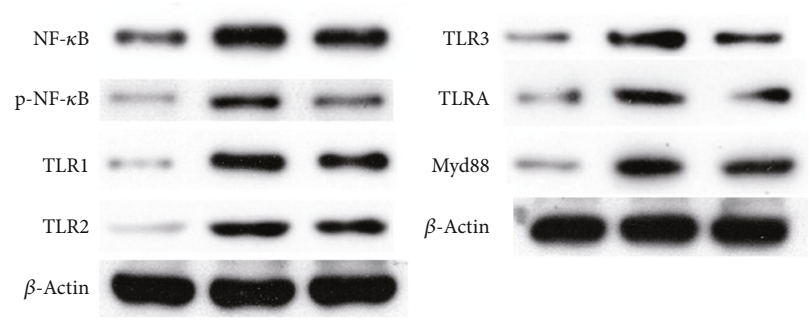

(g)

Figure 2: Continued. 

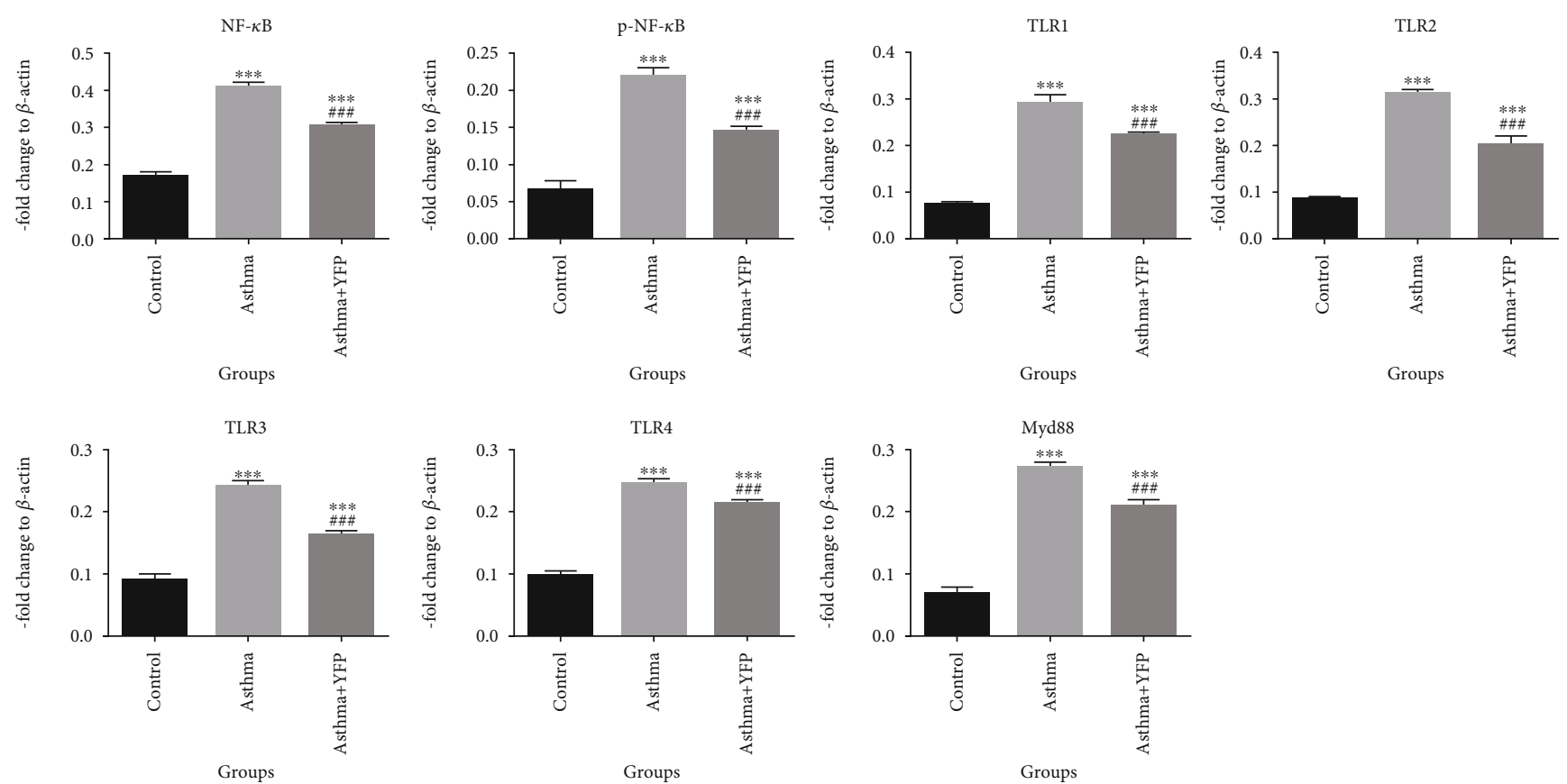

(h)

FIGURE 2: YFP promoted the inflammation in the lung of the mouse asthma model. (a) The BALF leukocyte count was checked by a hemocytometer. (b-f) The expression of TNF- $\alpha$, IL- $1 \beta$, IL-6, TGF- $\beta$, and IFN- $\gamma$ was determined by ELISA. (g, h) The expression of NF$\kappa \mathrm{B}, \mathrm{p}-\mathrm{NF}-\kappa \mathrm{B}$, TLR1, TLR2, TLR3, TLR4, and Myd88 was evaluated by Western blot. Data are presented as mean \pm SEM. ${ }^{* * *} P<0.001$ vs. control, ${ }^{\# \#} P<0.01$ vs. asthma, and ${ }^{\# \# \#} P<0.001$ vs. asthma.

of YFP (Figures 3(b)-3(d)). Besides, the expression of oxidative stress-related genes in the lung tissue was detected by qRT-PCR. The results are shown in Figures 3(e)-3(j). The gene expression level of GPX1, GPX4, CAT, SOD1, SOD2 and UCP2 was significantly suppressed in the asthma group, compared to the control group, and was promoted comparing with that by the asthma+YFP group $\left({ }^{* *} P<0.01\right.$ vs. control, ${ }^{* * *} P<0.001$ vs. control, and ${ }^{\sharp \# *} P<0.001$ vs. asthma).

3.4. YFP Ameliorated the Autophagy in the Lung of the Mouse Asthma Model. To evaluate the effects of YFP on autophagy in the lung tissues induced by asthma modeling, Western blot was used to determine the expression level of autophagyrelated proteins. As shown in Figure 4, ATG5, Beclin1, and LC3BII/I were significantly upregulated in asthma mice compared to control, which were greatly suppressed by the introduction of YFP $\left({ }^{* *} P<0.01\right.$ vs. control, ${ }^{* * *} P<0.001$ vs. control, and ${ }^{\# \# \# P} P<0.001$ vs. asthma).

3.5. The Distribution of Bacteria Species Was Slightly Changed by YFP in Asthma Mice. 16S rDNA sequencing analysis was performed to explore the effect of YFP on the gut microbiota of allergic asthma mice. As shown in Figure 5(a), the Venn diagram revealed that a total of 1045 distinct genera were identified upon YFP treatment. These observations indicated that the YFP treatment increased the bacterial diversity in the gut of the animal, although not statistically significant. Figures $5(\mathrm{~b})-5(\mathrm{~d})$ showed the microbial richness (Chaol) analysis, alpha diversity (Shannon) analysis, and PCoA analysis, respectively. However, no significant difference was observed between the asthma group and the YFP-treated group. As shown in Figure 5(e) and Table 2, the major difference in the relative abundance of the bacteria species in the feces of mice was observed between the control group and the asthma group, of which the top 15 species with the highest abundance were listed. Interestingly, except for Akkermansia (higher in the control group), the relative abundance of Prevotella, Oscillospira, Helicobacter, Coprococcus, Ruminococcus, Bacteroides, Flexispira, Odoribacter, and Turicibacter in the asthma mice was significantly elevated, compared to control $\left({ }^{*} P<0.05\right.$ vs. control). By the treatment of YFP, the relative abundance of Oscillospira, Helicobacter, Coprococcus, Ruminococcus, Flexispira, and Odoribacter was greatly decreased ( $P<0.05$ vs. asthma).

\section{Discussion}

Airway allergic inflammation is reported to be one of the pathological basis of allergic asthma [32]. Large amounts of inflammatory immune cells are recruited into the lung tissues when allergic occurs and release inflammatory cytokines to accelerate airway allergic inflammation, promoting aggravate allergic asthma process [33]. Therefore, it is of great importance to inhibiting airway inflammation in allergic asthma. In the present study, the antiasthma effect of YFP was investigated. HE staining showed that YFP ameliorated the lung injury caused by asthma modeling in mice. The tightjunction protein of lung tissues is closely related to the pathological state of allergic asthma, which is represented by the expression level of ZO-1, Claudin1, Claudin4, and Occludin 


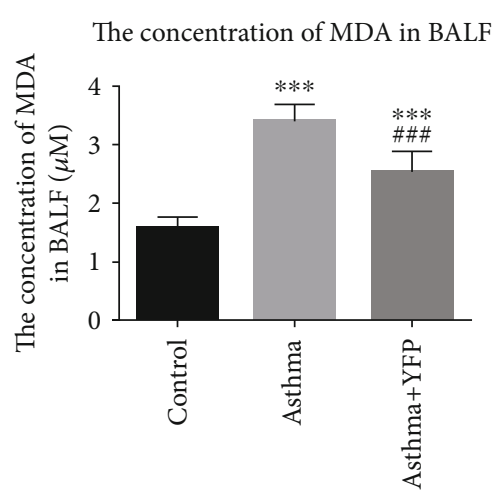

Groups

(a)

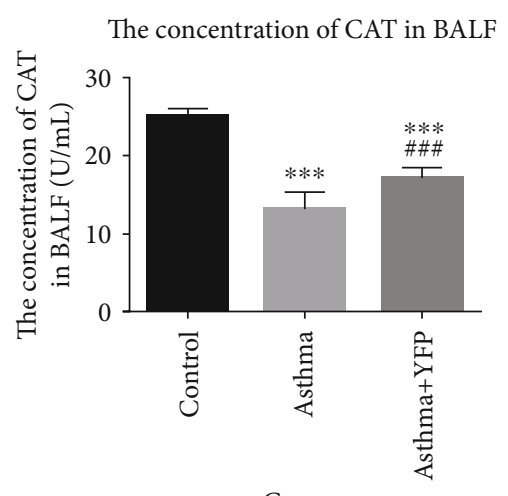

Groups

(d)

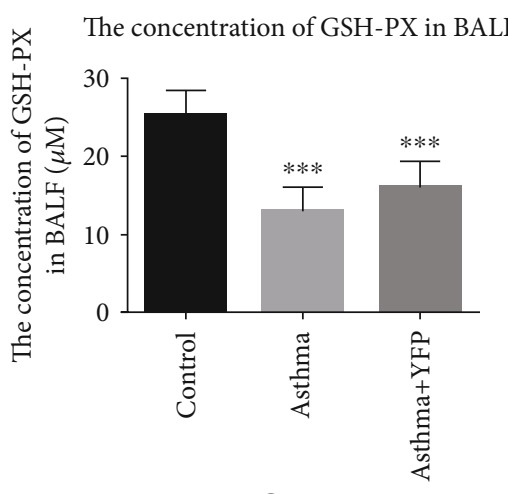

Groups

(b)

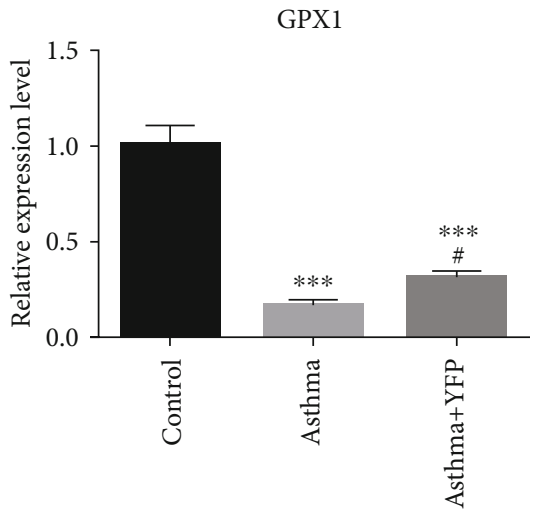

Groups

(e)

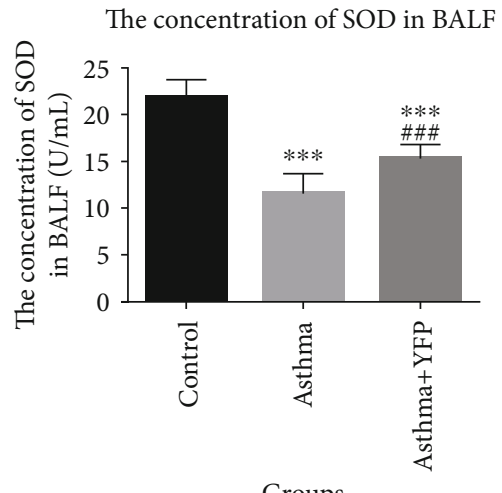

Groups

(c)

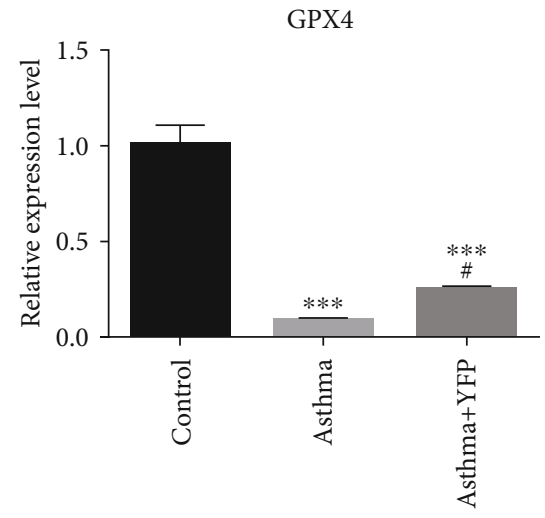

Groups

(f)

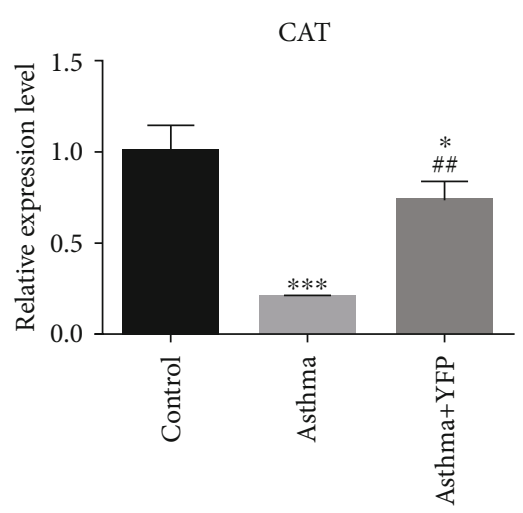

Groups

(g)

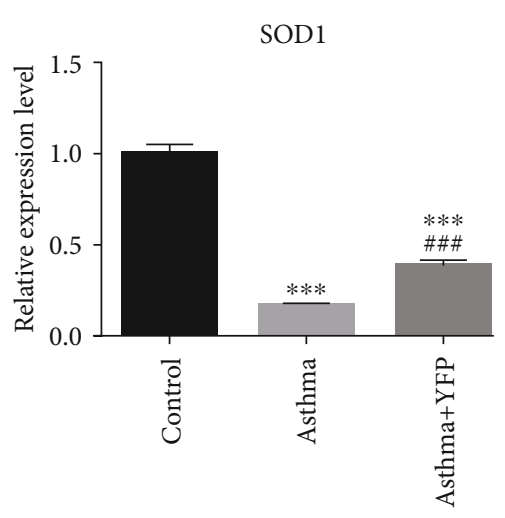

Groups

(h)

Figure 3: Continued. 


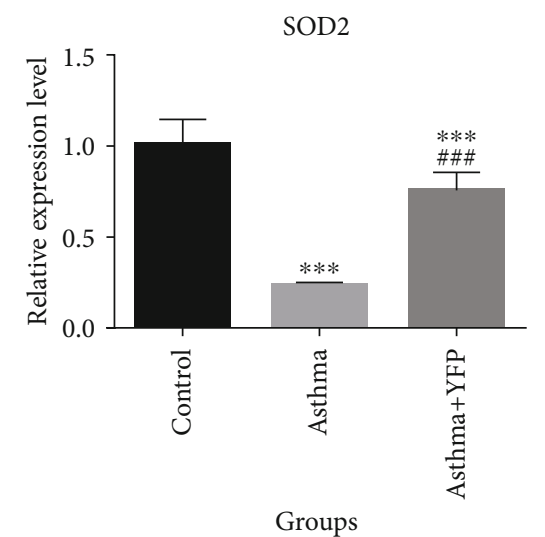

(i)

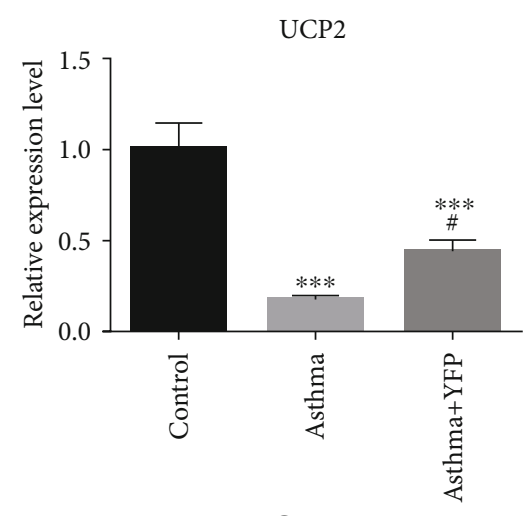

Groups

(j)

FIGURE 3: YFP ameliorated the level of oxidative stress in the lung of the mouse asthma model. (a-d) The concentration of MDA, GSH-Px, SOD, and CAT was determined by ELISA. (e-j) The gene expression level of GPX1, GPX4, CAT, SOD1, SOD2, and UCP2 was detected by qRT-PCR. Data are presented as mean \pm SEM. ${ }^{*} P<0.05$ vs. control, ${ }^{* * *} P<0.001$ vs. control, ${ }^{\#} P<0.05$ vs. asthma, ${ }^{\# \#} P<0.01$ vs. asthma, and $\# \#$ \# $<0.001$ vs. asthma.

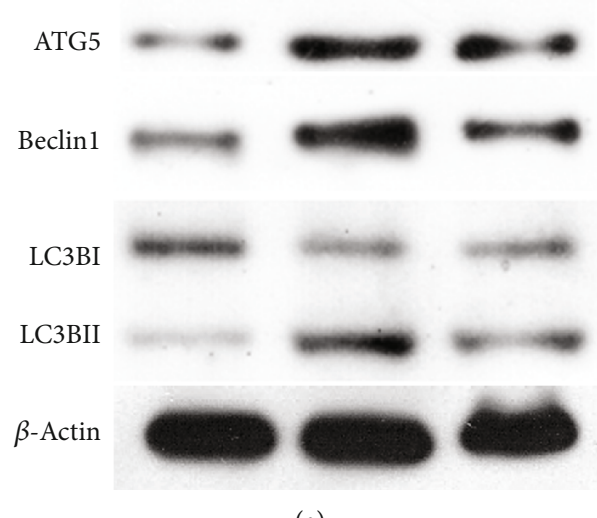

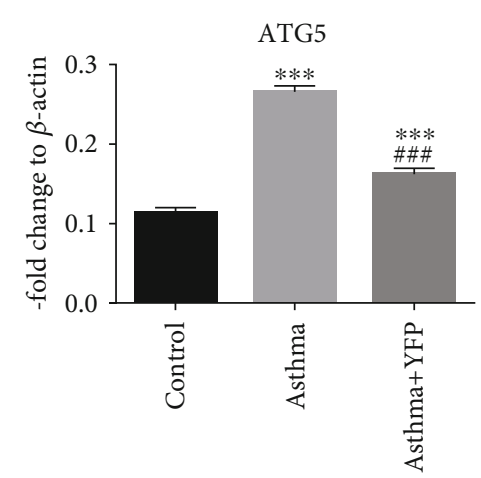

Groups

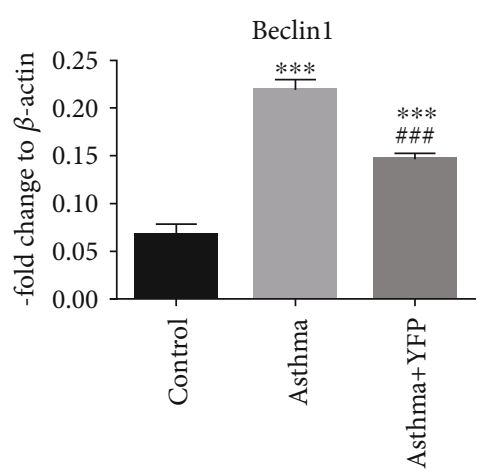

Groups

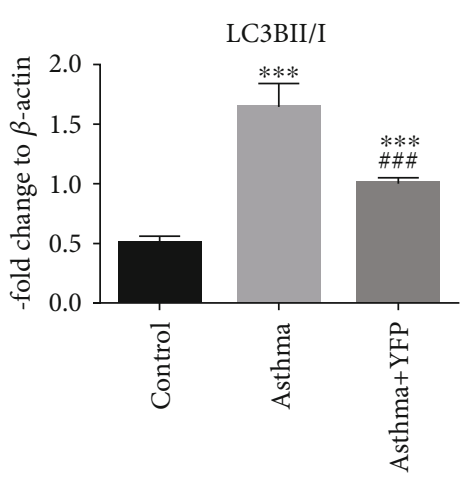

Groups

(b)

FIGURE 4: YFP ameliorated the level of oxidative stress in the lung of the mouse asthma model. (a) The expression level of ATG5, Beclin1, and LC3I/II detected by Western blot. (b) The quantitative results of the protein expression. Data are presented as mean \pm SEM. ${ }^{* *} P<0.01$ vs. control, ${ }^{* * *} P<0.001$ vs. control, and ${ }^{\# \# \#} P<0.001$ vs. asthma.

$[34,35]$. After the administration of YFP, the tight junction of lung tissue was greatly improved. The results indicated that the symptom of allergic asthma in mice was significantly improved by the administration of YFP. Besides, YFP pro- moted inflammation in the lung. By exploring the state of the inflammatory signal pathways in the lung tissues following treatments with YFP, we found that YFP greatly inhibited the TLR/NF- $\kappa$ B signal pathway in the lung tissue. These data 


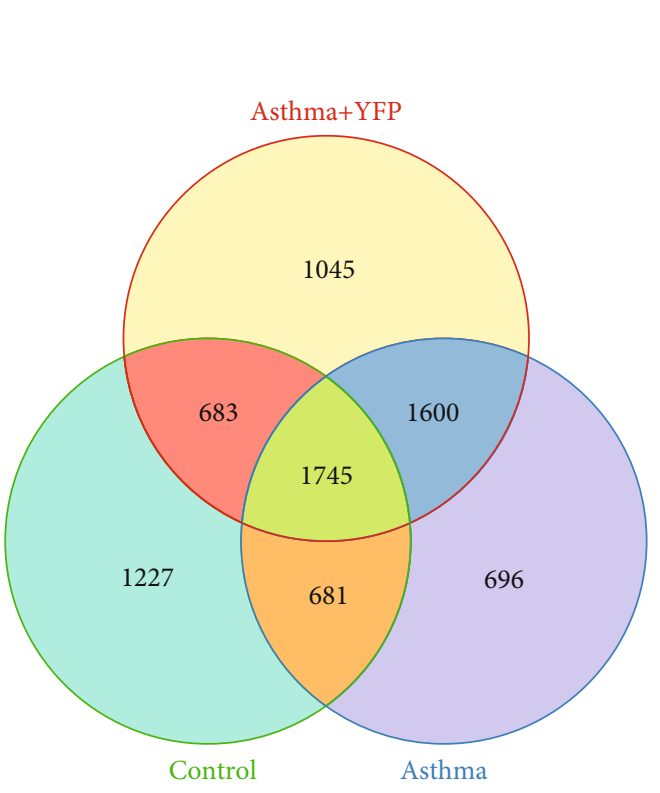

(a)

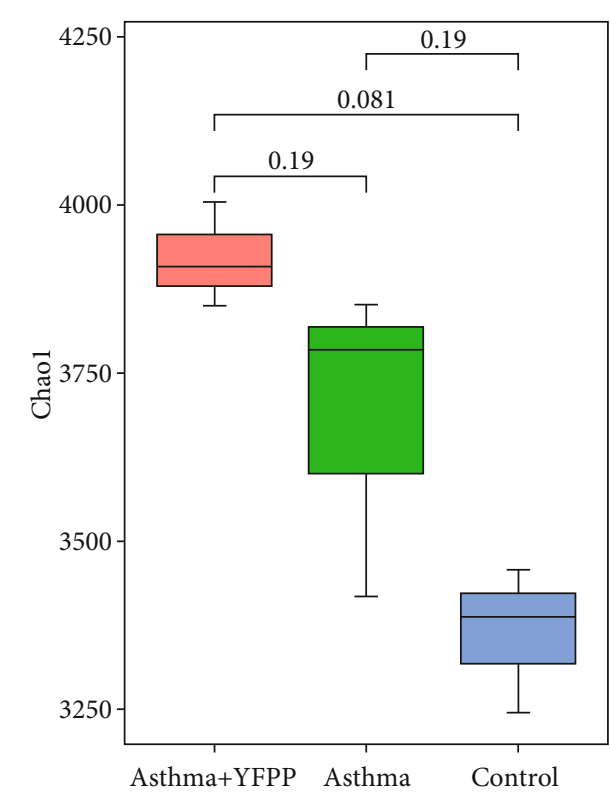

(b)
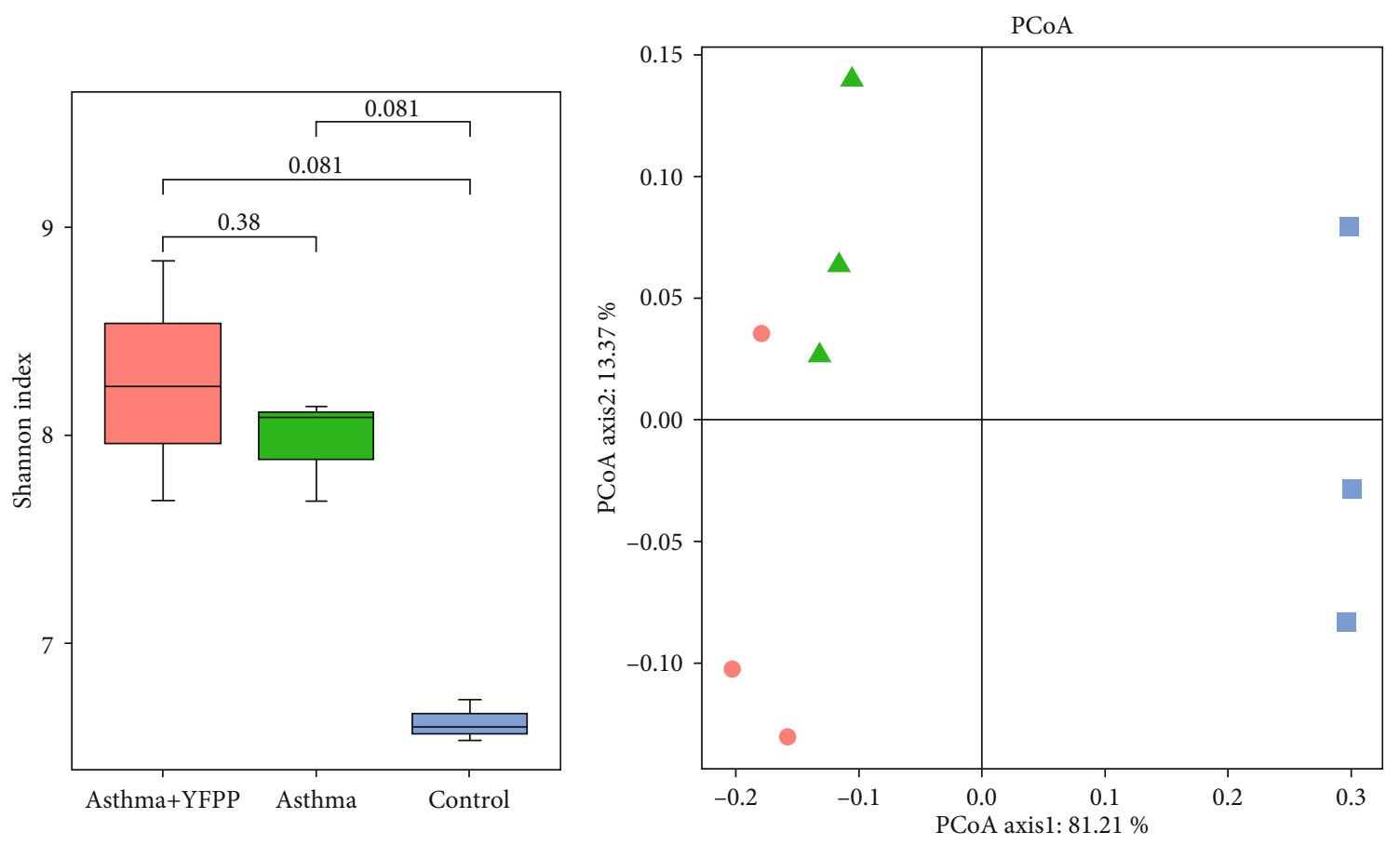

Group

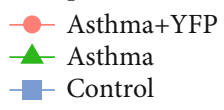

(c)

(d)

Figure 5: Continued. 


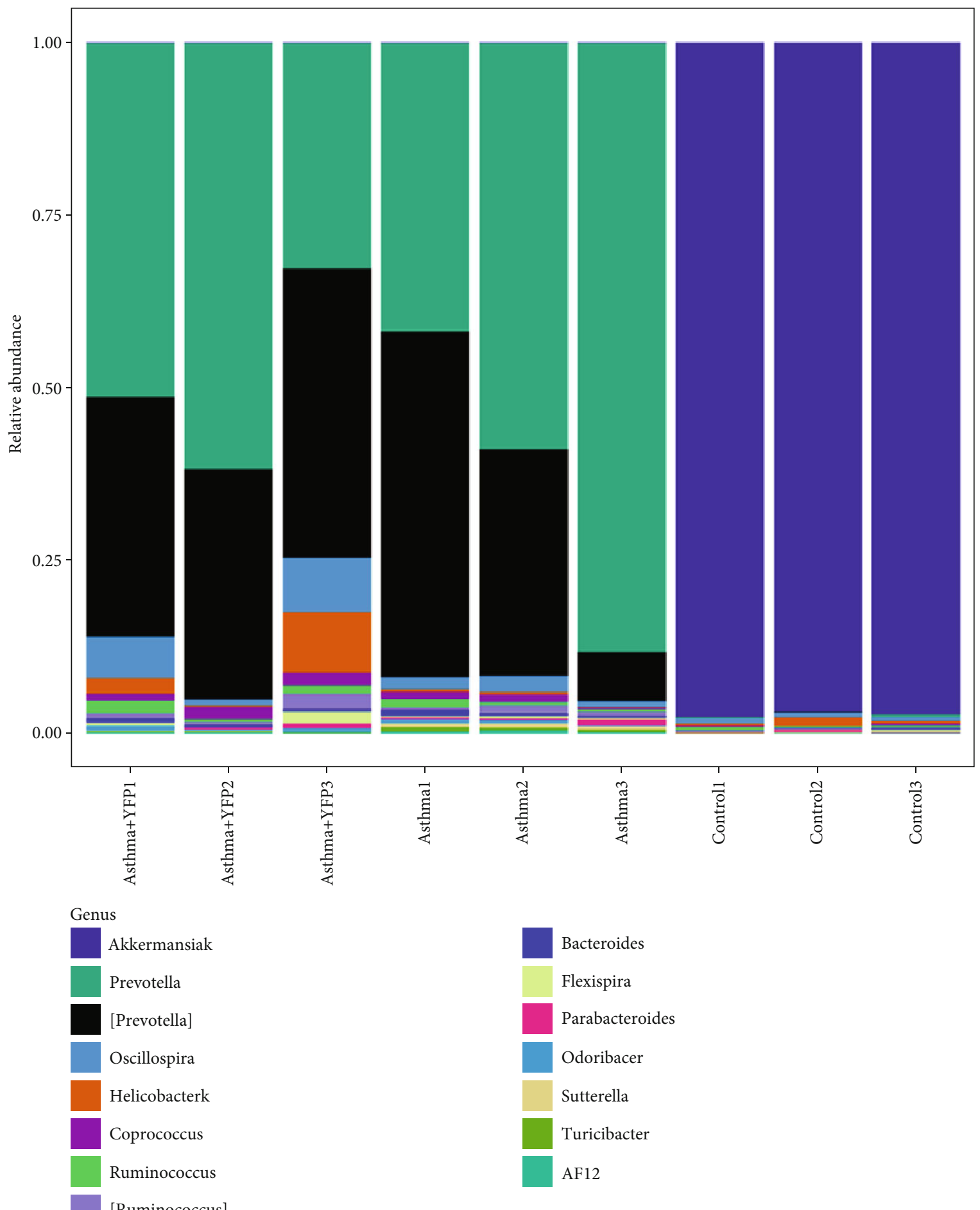

(e)

FIGURE 5: The distribution of gut microbiota was regulated by YFP. (a) Venn diagram of shared genus observed in the feces samples. (b) The microbial richness (Chao1) analysis in each group. (c) Alpha diversity (Shannon) analysis in each group. (d) The PCoA analysis in each group. (e) The histogram of species distribution of the top 15 with the highest relative abundance.

implied that the symptom of allergic asthma in the mouse model had been greatly improved by YFP, accompanied by suppression of the airway allergic inflammation.

To further investigate the possible mechanism underlying the inflammation inhibitory effects of YFP, the distribu- tion of gut microbiota in the feces of mice was explored. Salameh et al. [36] reported that gut microbiota plays a great role in the pathogenesis of allergic asthma, to which the inflammatory factors might be the mediators. In the present study, we found that although the relative abundance of the 
TABLE 2: The relative abundance of the top 15 bacteria species in the control, asthma, and YFP groups $\left({ }^{*} P<0.05\right.$ vs. control, ${ }^{\#} P<0.05$ vs. asthma).

\begin{tabular}{lccc}
\hline Name of genus & Relative abundance in the control group & Relative abundance in the asthma group & Relative abundance in the YFP group \\
\hline Akkermansiak & $0.376830 \pm 0.018474$ & $4.042394 \pm 1.664278^{*}$ & $7.308090 \pm 3.401218$ \\
Prevotella & $0.000692 \pm 0.000574$ & $0.151322 \pm 0.065380^{*}$ & $0.153308 \pm 0.083194$ \\
[Prevotella] & $7.146626 \pm 1.974750$ & $0.108791 \pm 0.008467^{*}$ & $0.066855 \pm 0.048877$ \\
Oscillospira & $0.002609 \pm 0.000602$ & $0.013902 \pm 0.009423^{*}$ & $0.003553 \pm 0.001185^{\#}$ \\
Helicobacterk & $0.002436 \pm 0.001818$ & $0.010009 \pm 0.010251^{*}$ & $0.000643 \pm 0.000375^{\#}$ \\
Coprococcus & $0.000674 \pm 0.000434$ & $0.004445 \pm 0.001347^{*}$ & $0.001724 \pm 0.000896^{\#}$ \\
Ruminococcus & $0.001198 \pm 0.000623$ & $0.003315 \pm 0.002597^{*}$ & $0.001678 \pm 0.001107^{\#}$ \\
[Ruminococcus] & $0.000406 \pm 9.398833$ & $0.003168 \pm 0.001985^{*}$ & $0.001569 \pm 0.000606^{\#}$ \\
Bacteroides & $0.000609 \pm 0.000204$ & $0.001355 \pm 0.000624^{*}$ & $0.001191 \pm 0.000658$ \\
Flexispira & $0.000674 \pm 0.000652$ & $0.001800 \pm 0.002127^{*}$ & $0.000515 \pm 0.000160^{\#}$ \\
Parabacteroides & $0.000714 \pm 0.000509$ & $0.000943 \pm 0.000621$ & $0.001299 \pm 0.001236$ \\
Odoribacter & $0 \pm 0$ & $0.001427 \pm 0.000976^{*}$ & $0.000848 \pm 0.000512^{\#}$ \\
Sutterella & $0.000279 \pm 0.000132$ & $0.000289 \pm 0.000168$ & $0.001239 \pm 0.000139$ \\
Turicibacter & $0 \pm 0$ & $0.000369 \pm 0.000155^{*}$ & $0.001182 \pm 0.000549^{\#}$ \\
AF12 & $0.000279 \pm 0.000139$ & $0.000472 \pm 0.000161$ & $0.000693 \pm 0.000180$
\end{tabular}

top 3 bacteria species was not reversed by YFP in the asthma mice, a significant inhibitory effect on the relative abundance of the top 4-8 bacteria species was achieved by the treatment of YFP, indicating a minor impact of YFP on the distribution of gut microbiota of the asthma mice. However, more evidence of the positive correlation between the antiasthma property of YFP and the change of gut microbiota distribution should be provided in our future work. In the present study, only one dosage of YFP was applied and we suspected that the change of gut microbiota distribution, such as the relative abundance of the bacteria species, $\alpha$-diversity index, and $\beta$-diversity index, might be enlarged if we increased the dosage, which will be verified in our subsequent work. Besides, in our future work, the inflammation profile both in the central nervous system and in the peripheral region will also be described to analyze the change of inflammation state before and after the treatment of YFP, which might bring direct evidence to explain the antiasthma property of YFP.

Oxidative stress is also a pathological manifestation of allergic asthma, the representative indexes of which are MDA, GSH-PX, SOD, and CAT [37]. In the present study, the oxidative stress level in the asthmatic mice was significantly inhibited by the treatment of YFP. Silveira et al. [21] reported that autophagy could induce eosinophil extracellular trap formation and allergic airway inflammation in a murine asthma model by activating oxidative stress. Tsai et al. [38] also reported that autophagy against oxidative stress-mediated apoptosis in normal and asthmatic airway epithelium is induced by complement regulatory protein CD46. In the present study, the expression level of autophagy-related proteins (ATG5, Beclin1, and LC3BII/I) was significantly suppressed by YFP. These data indicated that YFP might exert antiasthmatic effects by inhibiting oxidative stress through suppressing autophagy.
According to the data achieved in the present study, on the one hand, the inflammation induced in the murine asthma model could be alleviated by the treatment of YFP, which might be related to the regulatory effect of YFP on the TLR4/NF- $\kappa \mathrm{B}$ signaling pathway. In our future work, the specific inhibitor against the TLR4/NF- $\kappa \mathrm{B}$ signaling pathway will be introduced to verify the mechanism underlying the inhibitory effect of YFP against inflammation. On the other hand, oxidative stress in the lung tissues was ameliorated by YFP by regulating autophagy, which should also be verified by introducing the agonist of autophagy in our future work. As a result, the symptom of asthma in the murine model was significantly alleviated.

Collectively, our research showed that YFP might exert antiasthmatic effects by inhibiting airway allergic inflammation through regulating gut microbiota distribution and inhibiting oxidative stress levels through suppressing autophagy.

\section{Data Availability}

The data can be available if requested by the editor.

\section{Conflicts of Interest}

The authors declare there are no conflicts of interest regarding the publication of this paper.

\section{Acknowledgments}

This work was supported by the National Natural Scientific Fund of PR China (no. 81100059), the Natural Science Foundation of Hunan Province of China (2019JJ50849), and the Scientific Research Project of Science and Technology Office of Hunan Province (2018SK52505). 


\section{References}

[1] S. Hadebe and F. Brombacher, "Environment and host-genetic determinants in early development of allergic asthma: contribution of fungi," Frontiers in Immunology, vol. 10, p. 2696, 2019.

[2] W. Eder, M. J. Ege, and E. von Mutius, "The asthma epidemic," The New England Journal of Medicine, vol. 355, no. 21, pp. 2226-2235, 2006.

[3] M. M. Stein, C. L. Hrusch, J. Gozdz et al., "Innate immunity and asthma risk in Amish and Hutterite farm children," The New England Journal of Medicine, vol. 375, no. 5, pp. 411421, 2016.

[4] F. Hoffmann, F. Ender, I. Schmudde et al., "Origin, localization, and immunoregulatory properties of pulmonary phagocytes in allergic asthma," Frontiers in Immunology, vol. 7, p. 107, 2016.

[5] H. Vroman, I. M. Bergen, B. W. Li et al., "Development of eosinophilic inflammation is independent of B-T cell interaction in a chronic house dust mite-driven asthma model," Clinical and Experimental Allergy, vol. 47, no. 4, pp. 551-564, 2017.

[6] L. Fleming, S. Saglani, and A. Bush, "Asthma attacks: should we nail our colours to the mast (cell)?," The European Respiratory Journal, vol. 48, no. 5, pp. 1261-1264, 2016.

[7] C. Z. Han, I. J. Juncadella, J. M. Kinchen et al., "Macrophages redirect phagocytosis by non-professional phagocytes and influence inflammation," Nature, vol. 539, no. 7630, pp. 570574, 2016.

[8] Y. Zhang, Y. Xu, S. Liu et al., "Scaffolding protein Gab1 regulates myeloid dendritic cell migration in allergic asthma," Cell Research, vol. 26, no. 11, pp. 1226-1241, 2016.

[9] R. Divekar and W. J. Calhoun, "Heterogeneity of asthma in society," Advances in Experimental Medicine and Biology, vol. 795, pp. 31-41, 2014.

[10] Q. Ma, W. Huang, J. Zhao, and Z. Yang, "Liu Shen Wan inhibits influenza a virus and excessive virus-induced inflammatory response via suppression of TLR4/NF- $\kappa \mathrm{B}$ signaling pathway in vitro and in vivo," Journal of Ethnopharmacology, vol. 252, p. 112584, 2020.

[11] Y. W. Yu, M. X. Li, Z. Y. Zhang, and H. Yu, "The deficiency of CX3CL1/CX3CR1 system ameliorates high fructose dietinduced kidney injury by regulating NF- $\kappa \mathrm{B}$ pathways in CX3CR1-knock out mice," International Journal of Molecular Medicine, vol. 41, no. 6, pp. 3577-3585, 2018.

[12] R. Li, Y. Liu, L. Li, R. Zhang, and Y. Tang, "p120 inhibits LPS/TNF $\alpha$-induced endothelial Ang2 synthesis and release in an NF- $\kappa \mathrm{B}$ independent fashion," Cytokine, vol. 123, p. 154786, 2019.

[13] X. Tian, H. Zhao, Z. Zhang, Z. Guo, and W. Li, "Correction: intestinal mucosal injury induced by obstructive jaundice is associated with activation of TLR4/TRAF6/NF- $\kappa$ B pathways," PLoS One, vol. 14, no. 12, 2019.

[14] J. Hong, S. Li, D. Z. Markova et al., "Bromodomain-containing protein 4 inhibition alleviates matrix degradation by enhancing autophagy and suppressing NLRP 3 inflammasome activity in NP cells," Journal of Cellular Physiology, vol. 235, no. 7-8, pp. 5736-5749, 2020.

[15] H. Qi, J. Ren, L. Ba et al., "MSTN attenuates cardiac hypertrophy through inhibition of excessive cardiac autophagy by blocking AMPK /mTOR and miR-128/PPARgamma/NF-kappaB," Molecular Therapy-Nucleic Acids, vol. 19, pp. 507-522, 2019.
[16] Y. Suzuki, H. Maazi, I. Sankaranarayanan et al., "Lack of autophagy induces steroid-resistant airway inflammation," The Journal of Allergy and Clinical Immunology, vol. 137, no. 5, pp. 1382-1389.e9, 2016.

[17] P. Kuballa, W. M. Nolte, A. B. Castoreno, and R. J. Xavier, "Autophagy and the immune system," Annual Review of Immunology, vol. 30, no. 1, pp. 611-646, 2012.

[18] L. J. Martin, J. Gupta, S. S. Jyothula et al., "Functional variant in the autophagy-related 5 gene promotor is associated with childhood asthma," PLoS One, vol. 7, no. 4, 2012.

[19] D. L. Pham, S. H. Kim, P. Losol et al., "Association of autophagy related gene polymorphisms with neutrophilic airway inflammation in adult asthma," The Korean Journal of Internal Medicine, vol. 31, no. 2, pp. 375-385, 2016.

[20] A. Poon, D. Eidelman, C. Laprise, and Q. Hamid, “ATG5, autophagy and lung function in asthma," Autophagy, vol. 8, pp. 694-695, 2012.

[21] J. S. Silveira, G. L. Antunes, D. B. Kaiber et al., "Autophagy induces eosinophil extracellular traps formation and allergic airway inflammation in a murine asthma model," Journal of Cellular Physiology, vol. 235, pp. 267-280, 2020.

[22] I. Pinheiro, L. Robinson, A. Verhelst et al., "A yeast fermentate improves gastrointestinal discomfort and constipation by modulation of the gut microbiome: results from a randomized double-blind placebo-controlled pilot trial," BMC Complementary and Alternative Medicine, vol. 17, no. 1, p. 441, 2017.

[23] T. Pessi, Y. Sutas, A. Marttinen, and E. Isolauri, "Probiotics reinforce mucosal degradation of antigens in rats: implications for therapeutic use of probiotics," The Journal of Nutrition, vol. 128, no. 12, pp. 2313-2318, 1998.

[24] T. R. Klaenhammer, M. Kleerebezem, M. V. Kopp, and M. Rescigno, "The impact of probiotics and prebiotics on the immune system," Nature Reviews. Immunology, vol. 12, no. 10, pp. 728-734, 2012.

[25] C. A. Cuello-Garcia, J. L. Brozek, A. Fiocchi et al., "Probiotics for the prevention of allergy: a systematic review and metaanalysis of randomized controlled trials," The Journal of Allergy and Clinical Immunology, vol. 136, no. 4, pp. 952961, 2015.

[26] S. O. Jang, H. J. Kim, Y. J. Kim et al., "Asthma prevention by Lactobacillus rhamnosus in a mouse model is associated with CD4(+)CD25(+)Foxp3(+) T cells," Allergy, Asthma and Immunology Research, vol. 4, no. 3, pp. 150-156, 2012.

[27] S. Sagar, M. E. Morgan, S. Chen et al., "Bifidobacterium breve and Lactobacillus rhamnosus treatment is as effective as budesonide at reducing inflammation in a murine model for chronic asthma," Respiratory Research, vol. 15, no. 1, p. 46, 2014.

[28] X. Wang, Y. Hui, L. Zhao, Y. Hao, H. Guo, and F. Ren, “Oral administration of Lactobacillus paracasei L9 attenuates PM2.5-induced enhancement of airway hyperresponsiveness and allergic airway response in murine model of asthma," PLoS One, vol. 12, no. 2, 2017.

[29] J. Yu, S. O. Jang, B. J. Kim et al., "The effects of Lactobacillus rhamnosus on the prevention of asthma in a murine model," Allergy, Asthma and Immunology Research, vol. 2, no. 3, pp. 199-205, 2010.

[30] H. Wang, S. Li, H. Li, F. Du, J. Guan, and Y. Wu, "Mechanism of probiotic VSL\#3 inhibiting NF- $\kappa \mathrm{B}$ and TNF- $\alpha$ on colitis through TLR4-NF- $\kappa \mathrm{B}$ signal pathway," Iranian Journal of Public Health, vol. 48, no. 7, pp. 1292-1300, 2019. 
[31] R. Bhardwaj, B. P. Singh, N. Sandhu et al., "Probiotic mediated NF-kappaB regulation for prospective management of type 2 diabetes," Molecular Biology Reports, vol. 47, no. 3, pp. 23012313, 2020.

[32] G. Liu, M. A. Cooley, P. M. Nair et al., “Airway remodelling and inflammation in asthma are dependent on the extracellular matrix protein fibulin-1c," The Journal of Pathology, vol. 243, no. 4, pp. 510-523, 2017.

[33] Q. Zhang, L. Wang, B. Chen, Q. Zhuo, C. Bao, and L. Lin, "Propofol inhibits NF- $\kappa$ B activation to ameliorate airway inflammation in ovalbumin (OVA)-induced allergic asthma mice," International Immunopharmacology, vol. 51, pp. 158164, 2017.

[34] H. J. Kim, S. H. Lee, S. Jeong, and S. J. Hong, "Protease-activated receptors 2 -antagonist suppresses asthma by inhibiting reactive oxygen species-thymic stromal lymphopoietin inflammation and epithelial tight junction degradation," Allergy, Asthma \& Immunology Research, vol. 11, no. 4, pp. 560-571, 2019.

[35] H. T. Tan, S. Hagner, F. Ruchti et al., "Tight junction, mucin, and inflammasome-related molecules are differentially expressed in eosinophilic, mixed, and neutrophilic experimental asthma in mice," Allergy, vol. 74, no. 2, pp. 294-307, 2019.

[36] M. Salameh, Z. Burney, N. Mhaimeed et al., "The role of gut microbiota in atopic asthma and allergy, implications in the understanding of disease pathogenesis," Scandinavian Journal of Immunology, vol. 91, no. 3, 2019.

[37] S. Samarghandian, M. Azimi-Nezhad, T. Farkhondeh, and F. Samini, "Anti-oxidative effects of curcumin on immobilization-induced oxidative stress in rat brain, liver and kidney," Biomedicine \& Pharmacotherapy, vol. 87, pp. 223-229, 2017.

[38] Y. G. Tsai, Y. S. Wen, J. Y. Wang et al., "Complement regulatory protein $\mathrm{CD} 46$ induces autophagy against oxidative stress-mediated apoptosis in normal and asthmatic airway epithelium," Scientific Reports, vol. 8, no. 1, p. 12973, 2018. 